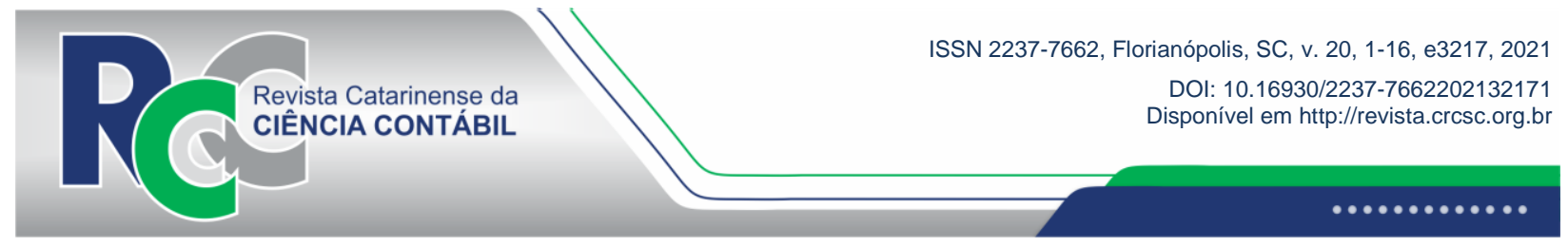

\title{
IMPLANTAÇÃO DAS NORMAS INTERNACIONAIS DE AUDITORIA PELOS TRIBUNAIS DE CONTAS BRASILEIROS
}

\section{IMPLEMENTATION OF INTERNATIONAL STANDARDS ON AUDITING BY THE BRAZILIAN COURTS OF AUDITORS}

\author{
TACIANA CAMILA WITCOSKI ESTEVAM \\ Universidade do Vale do Rio dos Sinos. \\ Endereço: Av. Dr. Nilo Peçanha, 1600 | Boa Vista | 91330-002 | \\ Porto Alegre/RS | Brasil. \\ (1) https://orcid.org/0000-0002-2978-721X \\ tacy.mila@gmail.com
}

\author{
JULIANA DANIELA RODRIGUES \\ Universidade do Vale do Rio dos Sinos. \\ Endereço: Av. Dr. Nilo Peçanha, 1600 | Boa Vista | 91330-002| \\ Porto Alegre/RS | Brasil. \\ (D) https://orcid.org/0000-0003-2061-2234 \\ julianarodrigs@yahoo.com.br
}

\section{LEANDRA DA SILVA}

Universidade do Vale do Rio dos Sinos.

Endereço: Av. Dr. Nilo Peçanha, 1600 | Boa Vista | 91330-002 |

Porto Alegre/RS | Brasil.

(D) https://orcid.org/0000-0003-2741-8397

leandrapoa@yahoo.com.br

\section{RESUMO}

O presente estudo objetivou analisar o processo de implantação das normas internacionais de auditoria pelos Tribunais de Contas (TCs) brasileiros, por meio de pesquisa exploratória e de levantamento, utilizando dois questionários com perguntas abertas e fechadas, que foram enviados a todos os TCs e às instituições que apoiam suas atividades. Os resultados da pesquisa demonstraram diferentes movimentos realizados pelos TCs na adesão às normas, em linha com pesquisas prévias sobre as práticas das Cortes de Contas brasileiras e das Entidades Fiscalizadoras Superiores internacionais. Dos 20 TCs respondentes (61\% do total), 60\% iniciaram o processo de implantação das normas, mas somente $10 \%$ concluíram-no. As normas internacionais de auditoria cuja implantação está mais avançada são as NBASP 20 e 30, que emanam os princípios da transparência e accountability com a gestão da ética pelos TCs. Os achados quanto à vasta disponibilidade de capacitação sobre o tema, ao apoio fornecido pelas organizações e à ausência de necessidade de utilização de recursos financeiros para a implantação pelos órgãos que concluíram o processo trazem dúvidas a respeito das motivações dos TCs que não iniciaram o processo. Embora os esforços verificados no sentido de qualificar a atuação dos TCs, parece haver

\footnotetext{
Editado em português e inglês. Versão original em português.

Recebido em 26/10/2021. Revisado em 6/12/2021. Aceito em 19/12/2021 pelos Prof. Dr. Sérgio Murilo Petri (Editor-Chefe) e Prof. Dr. Sandro Vieira Soares (Editor Adjunto). Publicado em 29/12/2021.

Copyright @ 2021 RCCC. Todos os direitos reservados. É permitida a citação de parte de artigos sem autorização prévia, desde que identificada a fonte.
} 
um longo caminho para a conclusão da implantação no país, devido à falta de previsão pela maioria dos respondentes.

Palavras-chave: Tribunal de Contas. Auditoria. NBASP. ISSAI. Implantação.

\begin{abstract}
This study aimed to analyze the process of implementation of international auditing standards by the Brazilian Courts of Auditors (TCs), through exploratory research and survey, using two questionnaires, with open and closed questions, sent to all TCs and institutions that support their activities. The survey results showed different movements made by the TCs in adhering to the rules, in line with previous research on the practices of Brazilian audit courts and of the Supreme Audit Institutions. Of the 20 TCs responding (61\% of the total), $60 \%$ started the process of implementing the standards, but only 10\% concluded. The international auditing standards whose implementation is more advanced are the NBASP 20 and 30, which bring the principles of transparency and accountability together with the management of ethics by the TCs. The findings regarding the wide availability of training on the subject, the support provided by organizations, and the absence of the need to use financial resources for implementation by the bodies that completed the process, raise doubts about the motivations of the TCs that did not initiate the process. Despite the efforts made to qualify the performance of the TCs, there seems to be a long way to go towards completing the implementation in the country due to the lack of forecast by most respondents.
\end{abstract}

Keywords: Court of Auditors. Audit. NBASP. ISSAI. Implementation.

\title{
1 INTRODUÇÃO
}

Nos últimos anos, em meio à divulgação de diversos casos de corrupção no Brasil, o trabalho dos órgãos de controle da Administração Pública tem recebido grande atenção. A atuação dos Tribunais de Contas (TCs), Entidades Fiscalizadoras Superiores (EFSs), é fundamental nesse contexto, pois tem o potencial de auxiliar na melhoria do desempenho governamental, no crescimento da democracia e no aumento da confiança dos cidadãos ao visar à qualidade da gestão dos recursos públicos (Gørrissen, 2020; Dragustin, Pitulice, \& Stefanescu, 2021).

Ao mesmo tempo, o setor público brasileiro tem passado por reformas no que diz respeito às normas e aos procedimentos utilizados, buscando adaptação a padrões internacionais. No caso da contabilidade, muitas ações ocorreram, recentemente, para a adoção das International Public Sector Accounting Standards (IPSAS), envolvendo esforços de todos os entes federativos, bem como os movimentos de coordenação conduzidos pelo Conselho Federal de Contabilidade (CFC) e pela Secretaria do Tesouro Nacional (STN), que iniciaram no ano de 2008 (STN, 2018).

Assim como na contabilidade, a auditoria do setor público possui organizações que apoiam suas atividades e influenciam o processo de convergência a novos padrões (Hay \& Cordery, 2020). Internacionalmente, a International Organization of Supreme Audit Institutions (INTOSAI) é a instituição que propõe as Normas Internacionais de Instituições Superiores de Auditoria (ISSAIs), e vem crescendo em legitimidade com mais de 70 anos de atuação (Reichborn-Kjennerud et al., 2019). No Brasil, a condução do processo de adoção das normas da INTOSAI tem sido realizada em parceria entre o Tribunal de Contas da União (TCU) e o Instituto Rui Barbosa (IRB), por meio da criação das Normas Brasileiras de Auditoria no Setor Público (NBASP) (TCU, 2020a).

A adoção de normas internacionais de auditoria no setor público pode promover diversos benefícios, assim, envolvendo maior percepção da qualidade do trabalho, controle mais eficaz dos recursos públicos e a geração de uma linguagem que favoreça o intercâmbio de experiências entre diversos países (López-Hernández, 2013). A utilização de padrões de auditoria, que sejam 
reconhecidos internacionalmente pelos TCs, constitui-se em importante instrumento de melhoria do controle e da gestão pública brasileira, gerando fortalecimento institucional (TCU, 2020b).

Tais benefícios tornam-se ainda mais relevantes no contexto de escândalos recentes, como os ganhos ilícitos por conselheiros do Tribunal de Contas do Estado do Rio de Janeiro (TCE-RJ) e a prevalência de más práticas organizacionais, como estudado por Lino e Aquino (2020). Outro fator que corrobora a necessidade de um padrão de atuação para os TCs são as diferentes interpretações nos julgamentos aplicados aos jurisdicionados, que têm dificultado a efetividade das regras de mitigação de riscos fiscais dos entes federativos (Nunes, Marcelino, \& Silva, 2019).

Contudo pesquisas recentes sugerem que a adoção de padrões internacionais aplicados à auditoria do setor público ainda é incipiente, verificando-se grandes divergências entre os procedimentos aplicados no caso dos TCs brasileiros (Azevedo \& Lino, 2018; Lino \& Aquino, 2018). Atualmente, não há obrigatoriedade de adoção das NBASP no país, e cada tribunal pode projetar movimentos distintos para a adoção das normas. Internacionalmente, conforme a literatura, apesar de haver muitos órgãos superiores de fiscalização associados à INTOSAI, a utilização de suas normas ainda está distante de alcançar a totalidade de seus membros (Kontogeorga, 2019; Manes-Rossi, Brusca, \& Condor, 2020; Cordery \& Hay, 2021).

Posto isso, considerando a importância do trabalho dos Tribunais de Contas para a qualidade da gestão pública brasileira e os benefícios advindos da adoção de padrões internacionais, surgiu o interesse de entender como cada órgão está atuando frente ao processo, a fim de identificar seus desafios, boas práticas e melhorias nas atividades de auditoria. Nesse sentido, este estudo teve como objetivo analisar a adoção das normas internacionais de auditoria do setor público pelos TCs brasileiros e o apoio prestado pelas organizações profissionais da área.

A literatura sobre auditoria no setor público ainda é escassa, o que surpreende dado que o setor representa grande parte das instituições auditadas (Hay \& Cordery, 2021). Ademais, apesar do crescente interesse em pesquisas acerca das diferentes práticas entre as entidades superiores de fiscalização, seja em esfera internacional (Manes-Rossi, Brusca, \& Condor, 2020; Cordery \& Hay, 2021), ou nos TCs brasileiros (Azevedo \& Lino, 2018; Lino \& Aquino, 2018; Lino \& Aquino, 2020; Nunes, Marcelino, \& Silva, 2019; Luz, Guarido, \& Sousa, 2021), não se teve conhecimento, no decorrer da presente pesquisa, de trabalhos que abordem o andamento do processo de implantação dos padrões internacionais pelos órgãos competentes.

Com este trabalho, busca-se preencher a lacuna no tema, especificamente no que diz respeito à adoção das normas internacionais de auditoria no setor público, para tanto, explorando o cenário do processo de convergência no Brasil e fornecendo uma base para análise da diversidade de movimentos e para suas possíveis causas. Adicionalmente, as informações trazidas neste estudo poderão ser úteis para o planejamento de ações de órgãos nacionais e internacionais no sentido de acelerar a adoção das normas, implementando ajustes para o crescimento da qualidade da fiscalização com escopo de melhor justificar receitas e gastos para a sociedade.

O estudo está organizado em cinco seções, incluindo a presente introdução. Na segunda seção, expõe-se a revisão teórica e empírica da temática das normas internacionais de auditoria do setor público. Na terceira seção são descritos os procedimentos metodológicos adotados na pesquisa. Na quarta seção, relatam-se os resultados e na quinta seção são apresentadas as considerações finais.

\section{AS ENTIDADES FISCALIZADORAS SUPERIORES E AS NORMAS DA INTOSAI}

As Entidades Fiscalizadoras Superiores (EFSs) são instituições antigas e tradicionais, com funcionamento disposto pela Constituição da jurisdição, evoluindo com o passar dos anos, mas refletindo cultura e tradições de longo prazo (Caruana \& Kowalczyk, 2021). Cada país costuma ter seu regulamento específico em torno da organização, das atividades e das responsabilidades de tais órgãos, assim, seguindo os reflexos contextuais (Manes-Rossi, Brusca, \& Condor, 2020). 
No Brasil, as entidades de fiscalização superiores são os TCs, que atuam no controle externo dos recursos públicos das três esferas de governo (Luz, Guarido, \& Sousa, 2021). O Tribunal de Contas da União (TCU) foi instituído de forma definitiva a partir da primeira Constituição republicana, em 1891 (Speck, 2000). Após, surgiram os TCs dos estados e dos municípios, existindo, atualmente, 33 órgãos no país.

As EFSs podem organizar-se como uma Corte de auditores com função judicial e poderes para impor sanções, caso dos TCs brasileiros e também de países como França, Itália e Portugal; podem ser colegiados sem funções judiciais nem poderes para impor sanções, caso da Alemanha e Holanda; e uma terceira forma é um auditor geral nomeado para dirigir escritórios de auditoria independentes, caso do Reino Unido e Dinamarca, ou escritórios fora da estrutura do governo, como na Finlândia (Caruana \& Kowalczyk, 2021).

A organização da auditoria aplicada ao setor público é fortemente baseada na visão de um contrato democrático, pois a existência de agentes executando auditorias que busquem assegurar a atuação do governo, de acordo com a legislação aplicada e os objetivos políticos, aumenta a credibilidade de que o trabalho em prol da sociedade será feito de uma maneira que atenda às suas expectativas (Gustavson \& Rothstein, 2013). Os auditores são encarregados de verificar se os recursos públicos estão sendo utilizados de forma responsável e efetiva, o que ajuda os entes governamentais em sua prestação de contas e na confiança dos cidadãos e demais stakeholders. Por conseguinte, uma boa auditoria é fundamental para evitar a má gestão dos recursos públicos (Manes-Rossi, Brusca, \& Condor, 2020).

As normas de auditoria utilizadas variam de acordo com o país, havendo os que aderem a normas internacionais, aplicáveis ao setor público ou ao setor privado, e os que adotam normas próprias, podendo estar baseadas nos padrões internacionais (Manes-Rossi, Brusca, \& Condor, 2020). Todavia os objetivos das auditorias conduzidas nos governos diferem dos objetivos da auditoria de outras organizações, dessa forma, limitando a utilização de normas que não sejam específicas (Azuma, 2008). Apesar das diferenças entre a auditoria no setor público e no setor privado, um ponto fundamental para sua qualidade é a independência do auditor, o que as normas buscam proteger. É evidente a importância de assegurar a integridade dos envolvidos para uma efetiva accountability e menores riscos de desvios de recursos (Caruana \& Kowalczyk, 2021).

As EFSs possuem corporações profissionais fortalecidas, que têm atuado além da representação de classe e estão inseridas na discussão acerca do aperfeiçoamento da atuação dos órgãos (Reichborn-Kjennerud et al., 2019; Hay \& Cordery, 2020; Luz, Guarido, \& Sousa, 2021). Atualmente, as normas de auditoria aplicadas ao setor público reconhecidas internacionalmente são editadas pela Organização Internacional das Entidades Fiscalizadoras Superiores (INTOSAI), que se classifica como uma dessas organizações de apoio.

A INTOSAI foi fundada em 1953, em um congresso que reuniu EFSs de 34 países, incluindo o Brasil (TCU, 2020). Atualmente, possui 195 países-membros, além de cinco organizações associadas e um país afiliado (INTOSAI, 2021). Sua atuação é independente, profissional e sem vinculação política, com objetivo de estabelecer apoio mútuo, intercâmbio de informações, atuar como representação internacional das Cortes de Contas e promover o fortalecimento das instituições com boa governança, desenvolvimento da atuação e normas de auditoria de qualidade para o setor público (INTOSAI, 2021).

As International Standards of Supreme Audit Institutions (ISSAIs) são originadas de trabalho iniciado em 2004, e em constante revisão, para a construção de capacidades e estruturas necessárias para organizar um conjunto de normas internacionais de auditoria do setor público (INTOSAI, 2021). A padronização proposta pela INTOSAI busca proporcionar homogeneidade e credibilidade às auditorias externas no setor público, assim, trazendo pontos como a qualidade dos trabalhos, credibilidade aos usuários, melhoria do processo, transparência, responsabilidade dos auditores, entre outros (Dragustin, Pitulice, \& Stefanescu, 2021). O marco referencial de normas profissionais da INTOSAI apresenta quatro níveis, conforme descrito pelo TCU (2020b): 
a) ISSAI Nível 1: trata dos princípios fundamentais.

b) ISSAI Nível 2: aborda os pré-requisitos relacionados ao funcionamento adequado das EFSs e à conduta de seus profissionais, tratando de pontos como independência, transparência e accountability, ética e controle de qualidade.

c) ISSAI Nível 3: oriundo da ISSAI 1 (Declaração de Lima) e das ISSAIs de nível 2, define a auditoria governamental e propõe a estrutura normativa de referência internacional.

d) ISSAI Nível 4: fornece diretrizes operacionais mais objetivas para retratar os princípios fundamentais de auditoria, permitindo a utilização nas rotinas de auditoria e no desenvolvimento de normas de auditoria.

As diferentes realidades políticas, sociais, culturais e econômicas das EFSs tornam desafiadora a existência de um modelo que seja adequado a todas elas (Caruana \& Kowalczyk, 2021, Cordery \& Hay, 2021). Nesse contexto de heterogeneidade, a INTOSAI incentiva que os países implementem as normas de acordo com seus contextos (INTOSAI, 2021). No Brasil, a primeira publicação das Normas Brasileiras de Auditoria no Setor Público (NBASP) se deu em 2015, partindo de uma convergência com base nas normas aprovadas pela INTOSAI e incluindo referências oriundas das Normas de Auditoria do Tribunal de Contas da União (NAT), das Normas de Auditoria Governamental (NAG), das resoluções da Associação dos Membros dos TCs do Brasil (Atricon), e da legislação vigente no país.

Em consonância com a importância das organizações de classe para a atuação das EFSs (Reichborn-Kjennerud et al., 2019; Hay \& Cordery, 2020; Luz, Guarido, \& Sousa, 2021), a iniciativa de edição das normas partiu do IRB, associação civil criada em 1973, com o objetivo de auxiliar os TCs no aperfeiçoamento e melhor desenvolvimento de suas atividades (IRB, 2020). O TCU também teve importante participação no processo com a tradução das normas da INTOSAI, que passaram por revisão pelo Subcomitê de Normas de Auditoria do IRB para a publicação das NBASP (TCU, 2020a). As NBASP estão organizadas em três níveis (IRB, 2019), que não possuem correspondência exata com os níveis apresentados pelas ISSAIs:

a) NBASP Nível 1 - Princípios fundamentais e pré-requisitos para o funcionamento dos TCs: relacionam-se com os níveis 1 e 2 da estrutura das ISSAIs vigente em 2013, com cinco normas que trazem os pré-requisitos organizacionais para os trabalhos de fiscalização.

b) NBASP Nível 2 - Princípios fundamentais de auditoria: relacionam-se com o nível 3 da estrutura das ISSAIs vigente em 2013. São quatro normas que envolvem os objetivos e o marco referencial das fiscalizações, por meio da definição dos elementos que devem estar presentes nos trabalhos, bem como os princípios a serem observados ao longo do processo.

c) NBASP Nível 3 - Requisitos mandatórios de auditoria: relacionam-se com o nível 4 da estrutura das ISSAIs vigente em 2013. Duas normas foram disponibilizadas em 2019 e transformaram os princípios que estão nas normas do nível anterior em requisitos mandatórios.

Não obstante a consolidação da edição das normas pelo IRB, desde 2015, a implantação e utilização efetiva pelos TCs precisa ocorrer para que possam ser observados os benefícios dos padrões internacionais de auditoria no país. A aplicação das novas normas pode não ser um trabalho simples e rápido, sendo necessária a suficiência de recursos humanos e materiais, bem como uma estratégia de implantação (López-Hernández, 2013). Apesar dos desafios apontados é escassa a literatura que se dedica a estudar o processo de adoção das normas da INTOSAI nacional e internacionalmente, e não se teve conhecimento, no decorrer da presente pesquisa, de estudos que abordem casos acerca da implantação, embora a existência de trabalhos que apontam a falta de harmonização de práticas de auditoria.

Estudos recentes se dedicam a comparar as diferentes práticas utilizadas pelos TCs brasileiros, evidenciando a ausência de padronização tanto entre diferentes jurisdições quanto nos trabalhos dos auditores de um mesmo órgão (Azevedo \& Lino, 2018; Lino \& Aquino, 2018; Lino \& Aquino, 2020; Nunes, Marcelino, \& Silva, 2019; Luz, Guarido, \& Sousa, 2021). Ademais, a 
baixa utilização e o pouco conhecimento das NBASP foram encontrados em trabalho empírico de Azevedo e Lino (2018), no qual são assinaladas possíveis dificuldades de implantação das normas.

No cenário internacional também são verificadas diferenças. Cordery e Hay (2021), em pesquisa survey respondida por EFSs de países integrantes da INTOSAI, encontraram que 55\% utilizavam as ISSAIs, enquanto as demais utilizavam referências distintas. A harmonização em torno das normas internacionais de auditoria também não é verificada mesmo em uma região, como é o caso da Europa, que foi estudado por Manes-Rossi, Brusca e Condor (2020). Ademais, mesmo com o acelerado desenvolvimento dos padrões de auditoria, muitas EFSs têm enfrentado desafios para a implantação de melhorias em seus procedimentos (Gørrissen, 2020).

Dada a importância da atuação dos TCs para a boa aplicação dos recursos públicos e seu consequente impacto no bem-estar da população brasileira, a busca pela qualificação de suas atividades pela utilização dos padrões internacionais foi analisada para o presente trabalho, cujos procedimentos metodológicos são descritos na seção seguinte.

\section{PROCEDIMENTOS METODOLÓGICOS}

A presente pesquisa classifica-se como exploratória, de levantamento e qualitativaquantitativa. Os procedimentos metodológicos estão descritos na Figura 1. Para analisar a implantação das normas internacionais de auditoria no Brasil, a população estudada envolveu os 33 TCs existentes: um TCU, 27 TCs estaduais, três TCs dos municípios e dois TCs municipais, e sete organizações que apoiam as atividades de auditoria na Administração Pública: Associação Brasileira dos Tribunais de Contas dos Municípios (Abracom), Associação dos Membros dos Tribunais de Contas do Brasil (Atricon), Associação Nacional dos Ministros e ConselheirosSubstitutos dos Tribunais de Contas (Audicon), Conselho Nacional de Presidentes dos Tribunais de Contas (CNPTC), Conselho Federal de Contabilidade (CFC), Instituto dos Auditores Independentes do Brasil (Ibracon) e Instituto Rui Barbosa (IRB), dada a relevância de tais entidades para o fortalecimento do trabalho dos TCs evidenciada pela literatura do tema (Reichborn-Kjennerud et al., 2019; Hay \& Cordery, 2020; Luz, Guarido, \& Sousa, 2021).

A coleta de dados foi realizada por meio de questionários encaminhados via Portal de Acesso à Informação e/ou via e-mail, no período de 6 a 12 de setembro de 2020. O recebimento das respostas ocorreu no período de 10 de setembro a 27 de outubro de 2020. Das 40 instituições consultadas, 26 responderam ao questionário, com a amostra totalizando $65 \%$ do total, sendo seis órgãos apoiadores e 20 TCs. Entre os que não responderam, houve dois indeferimentos dos pedidos justificando que não colaboram com pesquisas acadêmicas por regras internas; dois indeferimentos alegando algum tipo de problema de cadastro e, apesar do envio de recurso, não houve retorno; três confirmações de recebimento sem retorno; e os demais não confirmaram o recebimento.

\begin{tabular}{|c|c|c|}
\hline Etapa 1 - Elaboração dos Questionários & Etapa 2 - Coleta de Dados & Etapa 3 - Análise das Respostas \\
\hline Q1 - Organizações apoiadoras & Envio & Análise qualitativa \\
\hline $\begin{array}{l}\text { - Questões abertas: forma de apoio, motivação, } \\
\text { desafios, benefícios, cronograma, recursos, } \\
\text { atuação conjunta (López-Hernández, 2013; } \\
\text { Gørrissen, 2020). }\end{array}$ & $\begin{array}{l}\text { Envio aos } 33 \text { TCs e r sete } \\
\text { organizações apoiadoras r via } \\
\text { Portal de Acesso à Informação } \\
\text { e/ou } e \text {-mail }(6 \text { a } 12 / 09 / 2020) \text {. }\end{array}$ & $\begin{array}{l}\text { Técnica de análise de conteúdo } \\
\text { Bardin (2011), com categorias } \\
\text { construídas de acordo com as } \\
\text { respostas das questões abertas. }\end{array}$ \\
\hline Q2 - Tribunais de Contas & Retorno & Análise quantitativa \\
\hline $\begin{array}{|lr|}\text { - Questão fechada: situação geral implantação. } \\
\text { - Questões abertas contextualizadas ao status do } \\
\text { processo: motivação, desafios, recursos, } \\
\text { normativo, prazo, benefícios } \\
\text { Hernández, 2013; Gørrissen, 2020). } \\
\text { - Questão fechada sobre situação de cada norma. } \\
\end{array}$ & $\begin{array}{l}\text { Recebimento de respostas de } \\
\text { seis órgãos apoiadores e } 20 \text { TCs } \\
(10 / 09 \text { a 27/10/2020). }\end{array}$ & \\
\hline
\end{tabular}

Figura 1. Procedimentos Metodológicos.

Fonte: elaborada pelas autoras. 
Foram confeccionados dois diferentes questionários que, ao longo da apresentação dos resultados, são identificados como Questionário 1 (Q1), específico para as instituições que apoiam o financeiro e a auditoria do setor público brasileiro, e Questionário 2 (Q2), específico para os TCs. Ambos os instrumentos contêm questões abertas abordando como estão apoiando/conduzindo o processo, quais suas motivações, desafios e benefícios esperados, e como se estão organizando em termos de formalização, cronogramas e recursos humanos e financeiros, pontos relatados pela literatura como necessários à implantação das normas (López-Hernández, 2013, Gørrissen, 2020).

O Q2 inicia com uma questão principal fechada que indaga a atual situação da implantação das normas internacionais de auditoria no TC, isto é, se foi iniciada, se está sendo estudada, se está em andamento ou se foi concluída. Para cada uma das opções, relacionaram-se perguntas abertas contextualizadas ao status do processo no órgão, abordando os pontos trazidos no parágrafo anterior, além de questões fechadas concernentes à situação da implantação de cada uma das normas para os casos nos quais os trabalhos foram iniciados ou concluídos.

Na primeira fase da pesquisa, os questionários foram submetidos à validação de face $\mathrm{e}$ conteúdo por especialistas no tema, sendo analisados por três auditores que atuam em TCs do país. Diante dos apontamentos recebidos, os questionários foram reformulados para alcançar melhor eficácia na aplicação. As respostas recebidas foram analisadas de forma quantitativa, por meio da análise de frequência das respostas às perguntas fechadas, e qualitativa, por meio da técnica de análise de conteúdo (Bardin, 2011), com categorias construídas de acordo com as respostas para cada ponto analisado pelas questões abertas, conforme o apresentado nas tabelas da seção 4 .

\section{ANÁLISE DOS RESULTADOS}

\subsection{Implantação das normas internacionais de auditoria nos Tribunais de Contas}

Além do estágio de implantação de cada uma das normas nos tribunais, é importante conhecer as medidas tomadas por cada órgão para a adequação aos padrões internacionais. Dada a relevância das organizações profissionais para as atividades das entidades fiscalizadoras superiores (Reichborn-Kjennerud et al., 2019; Hay \& Cordery, 2020; Luz, Guarido, \& Sousa, 2021), as ações realizadas pelas instituições apoiadoras nesse processo também foram analisadas.

Dessa forma, para as instituições apoiadoras (Q1), solicitaram-se informações sobre como estão atuando na promoção da utilização das NBASP, o principal motivo que levou a instituição a atuar no processo de implantação, quais órgãos têm atuado conjuntamente para apoiar o processo da adoção, como estão ocorrendo essas interações, quais recursos financeiros foram ou seriam utilizados para dar início ao processo de implantação e a possível existência de um cronograma para orientação do processo em todos os TCs. Essas respostas são elencadas na Tabela 1.

Os resultados indicaram que o apoio prestado para a implantação das normas reside principalmente na oferta de capacitação e orientação, ponto condizente com a literatura (Reichborn-Kjennerud et al., 2019; Hay \& Cordery, 2020). Foi verificada atuação conjunta entre organizações nacionais e internacionais, e que as motivações de apoio ao processo estavam de acordo com a qualificação e legitimação (Cordery \& Hay, 2021).

Com relação aos recursos financeiros utilizados, nenhuma das instituições apoiadoras apresentou gastos. Quanto à existência de um cronograma para coordenação das atividades, foi informada, por uma das organizações, a existência de cronograma com previsão de conclusão da convergência em 2021, o que, dada a proximidade de data e a situação atual verificada no estudo, possivelmente, não será alcançado por todos os TCs. 


\section{Tabela 1}

\section{Respostas das instituições apoiadoras referentes à implantação das normas internacionais} de auditoria nos Tribunais de Contas

\begin{tabular}{|c|c|c|}
\hline Questão & $\begin{array}{c}\text { N. }{ }^{\circ} \text { de } \\
\text { respostas }\end{array}$ & Motivos apontados pelas instituições \\
\hline $\begin{array}{l}\text { Incentivo à utilização } \\
\text { das NBASP }\end{array}$ & 2010 & $\begin{array}{l}\text { Promovem, participam ou oferecem cursos, fóruns e seminários com o } \\
\text { intuito de divulgar as NBASP e orientam sobre auditoria em áreas } \\
\text { específicas. }\end{array}$ \\
\hline \multirow{4}{*}{$\begin{array}{c}\text { Motivação para o apoio } \\
\text { ao processo de } \\
\text { implantação das } \\
\text { normas }\end{array}$} & 3 & $\begin{array}{l}\text { Aceitação dos trabalhos de auditoria em qualquer jurisdição e alinhamento } \\
\text { com os principais organismos nacionais e internacionais de auditoria. }\end{array}$ \\
\hline & 1 & $\begin{array}{l}\text { Qualificação dos trabalhos da auditoria e, como consequência, melhoria } \\
\text { do atendimento e na confiança da população. }\end{array}$ \\
\hline & 1 & $\begin{array}{l}\text { Posição central de coordenação, ocupada pela instituição, perante o } \\
\text { sistema. }\end{array}$ \\
\hline & 1 & Não respondeu. \\
\hline \multirow{3}{*}{$\begin{array}{l}\text { Instituições envolvidas } \\
\text { e forma de interação no } \\
\text { apoio }\end{array}$} & 4 & $\begin{array}{l}\text { Abracom, Atricon, Audicon, CNPTC, CFC, Ibracon e IRB. A interação } \\
\text { entre os órgãos ocorre cotidiana e harmonicamente nas diversas ações } \\
\text { promovidas, na maioria dos casos, como único órgão. }\end{array}$ \\
\hline & 1 & $\begin{array}{l}\text { Intosai, IIA (The Institute of Internal Auditors), Banco Mundial, IFAC } \\
\text { (International Federation of Accountants). }\end{array}$ \\
\hline & 1 & Não respondeu. \\
\hline \multirow{3}{*}{$\begin{array}{c}\text { Quais recursos } \\
\text { financeiros foram ou } \\
\text { serão utilizados para } \\
\text { iniciar o processo }\end{array}$} & 4 & $\begin{array}{l}\text { O custeio da implantação partiria do IRB, com a destinação de parte do } \\
\text { orçamento por cada um dos TCs. }\end{array}$ \\
\hline & 1 & Participação indireta, não teve ciência dos gastos. \\
\hline & 1 & Não respondeu. \\
\hline \multirow{2}{*}{$\begin{array}{c}\text { Existência de } \\
\text { cronograma com início } \\
\text { e fim da implantação }\end{array}$} & 5 & $\begin{array}{l}\text { Não foi confeccionado cronograma, pois cada TC dever-se-ia organizar } \\
\text { internamente. }\end{array}$ \\
\hline & 1 & $\begin{array}{l}\text { Para a primeira fase do processo de convergência de todas as NBASP, } \\
\text { existe um cronograma indicando } 2021 \text { para essa conclusão. }\end{array}$ \\
\hline
\end{tabular}

Fonte: elaborada com dados das respostas ao Q1 da pesquisa.

No questionário enviado aos TCs (Q2), as perguntas foram confeccionadas com base na atual situação do processo de implantação das NBASP no órgão. Foram recebidas respostas de $60 \%$ (20) dos 33 TCs, e as perguntas partiram da situação do processo de implantação das normas internacionais de auditoria. A Figura 2 ilustra a situação do processo no momento da pesquisa.

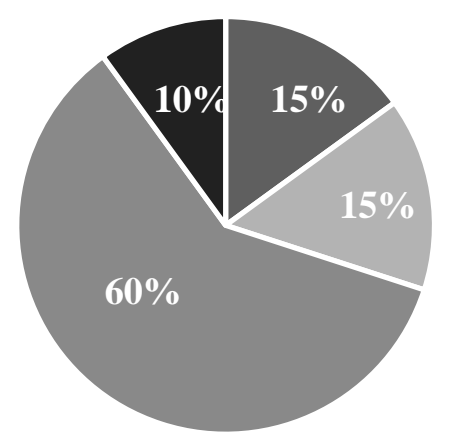

- Não iniciado

- Sendo estudado

- Em andamento

- Concluído

Figura 2. Situação do processo de implantação das NBASP nos Tribunais de Contas Fonte: elaborada com dados das respostas ao Q2 da pesquisa.

Destaca-se que $15 \%$ (3) dos TCs da amostra informaram que ainda não iniciaram o processo de implantação das normas, e 15\% (3) encontravam-se em estágio inicial de estudos, o que permite inferir que $30 \%$ (6) ainda não utilizavam os padrões internacionais. A maior parte dos 
respondentes informou que a implantação estava em andamento (12, ou $60 \%$ da amostra) e somente $10 \%$ (2) concluíram o processo. Assim, verificou-se que 70\% (14) dos TCs respondentes observavam, ao menos, parte das normas internacionais de auditoria. A heterogeneidade dos movimentos observados estava coerente com pesquisas recentes sobre as diferentes práticas dos TCs brasileiros (Azevedo \& Lino, 2018; Lino \& Aquino, 2018; Lino \& Aquino, 2020; Nunes, Marcelino, \& Silva, 2019; Luz, Guarido \& Sousa, 2021) e das EFSs internacionais (Manes-Rossi, Brusca, \& Condor, 2020; Cordery \& Hay, 2021).

Com base na primeira análise, são apresentados os resultados para cada uma das situações do processo de atendimento às normas, considerando que, para cada status, há uma quantidade de questões diferentes, visto que quanto mais próximo da conclusão do processo, mais informações podem ser colhidas. A Tabela 2 apresenta as medidas tomadas por cada TC para a implantação.

A Tabela 2 exibe os resultados para os três TCs (15\%) que informaram que a adoção das NBASP não foi iniciada. Questionaram-se os principais motivos pelos quais o órgão não iniciou o processo e se há previsão para o começo dos trabalhos. Os TCs informaram que, até aquele momento, não havia data para o início, relataram, dificuldades como a falta de determinação da presidência e a necessidade de recursos humanos e materiais. Os resultados foram condizentes com os desafios à implantação das normas apontados na literatura, sublinhando a importância da liderança e da capacidade organizacional (López-Hernández, 2013, Gørrissen, 2020).

A segunda situação relata os resultados para os três TCs (15\%) que informaram que a adoção das normas estava sendo estudada. Para essa situação, questionou-se a existência de profissionais envolvidos na avaliação da possibilidade de iniciar o processo, se houve estudo sobre a necessidade da utilização de recursos financeiros e as principais objeções que impediam o início da implantação das NBASP. De acordo com as respostas, dois tribunais já organizaram recursos humanos para o projeto, mas encontraram como obstáculos a pandemia de Covid-19 (2) e a ausência de normativo interno determinando a adesão às normas (1).

A terceira situação exibida voltou-se para os 12 TCs (60\%) que estavam com o processo de implantação em andamento. Questionou-se, então, o principal motivo que levara a iniciar o processo, a quantidade de profissionais alocados, a necessidade de recursos financeiros, se o tribunal possuía um normativo tratando dessa adesão, o tempo que levariam para concluir a implantação das normas e quais tinham sido implantadas pelo órgão. As informações quanto à motivação para o início do processo de adoção são um dos objetivos técnicos do IRB, que diz que a utilização das NBASP consiste na "essencialidade das normas profissionais para a credibilidade, qualidade e profissionalismo da auditoria do setor público" (IRB, 2020). Até o final de 2021, conforme prazo apontado em cronograma referido por uma das instituições apoiadoras, mais dois TCs deveriam concluir a implantação. Destaca-se que o tempo estimado para a conclusão não foi respondido por dez tribunais, o que levou a entender que a implantação das NBASP não é algo fácil e rápido de se realizar (López-Hernández, 2013; Kontogeorga, 2019; Gørrissen, 2020).

A quarta situação apresentada é a dos dois TCs (10\%) que já concluíram o processo de implantação das normas. Para essa situação, questionou-se o motivo que levara o órgão a iniciar o processo, a quantidade de profissionais alocados, a necessidade de recursos financeiros, se havia normativo tratando da adesão e o tempo gasto para a conclusão. A principal motivação citada foi a importância da padronização para a qualidade dos trabalhos.

Foram verificados indícios no que tange à importância da existência de um normativo interno tratando da adesão às normas, visto que foi bastante observado nos TCs em andamento (9) ou com as normas concluídas (2), enquanto sua inexistência foi apresentada como um obstáculo por um dos TCs que não iniciaram o processo. A causa para tanto pode estar relacionada ao formalismo da Administração Pública, mas também à importância da liderança e do planejamento para o sucesso da adoção (López-Hernández, 2013; Gørrissen, 2020). Já a utilização de recursos materiais, alegada como dificultador pelos TCs que não iniciaram a implantação das normas, não 
foi intensiva pelos TCs que estão avançando no processo, restando dúvidas sobre a relevância de sua necessidade para a implantação, pelo menos, em alguns contextos.

Tabela 2

\section{Medidas tomadas por cada TC para implantação das normas internacionais de auditoria}

\begin{tabular}{|c|c|c|c|}
\hline Situação & Questão & $\begin{array}{l}\mathrm{N}^{\circ} \text { de } \\
\text { respostas }\end{array}$ & Respostas dos tribunais \\
\hline \multirow{2}{*}{ 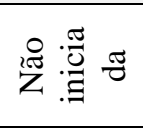 } & $\begin{array}{l}\text { Principais } \\
\text { motivos }\end{array}$ & 3 & $\begin{array}{l}\text { Falta de determinação da presidência, dificuldades com sistema de } \\
\text { informação, conhecimento, capacitação e carência de corpo técnico. }\end{array}$ \\
\hline & Previsão & 3 & Não há previsão. \\
\hline \multirow{5}{*}{ 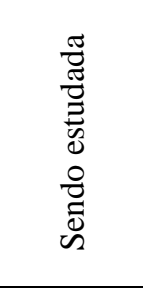 } & \multirow{2}{*}{$\begin{array}{l}\text { Recursos } \\
\text { humanos }\end{array}$} & 1 & Não houve a nomeação formal de equipe para condução do projeto. \\
\hline & & 2 & Houve nomeação de servidores para os estudos. \\
\hline & $\begin{array}{l}\text { Recursos } \\
\text { financeiros }\end{array}$ & 3 & Não houve ainda a estimativa de gastos para esse processo. \\
\hline & \multirow{2}{*}{$\begin{array}{c}\text { Obstáculos } \\
\text { ao } \\
\text { processo }\end{array}$} & 2 & Pandemia de Covid-19. \\
\hline & & 1 & $\begin{array}{l}\text { Ausência de normativo interno determinando o alinhamento do TC às } \\
\text { NBASP. }\end{array}$ \\
\hline \multirow{16}{*}{ 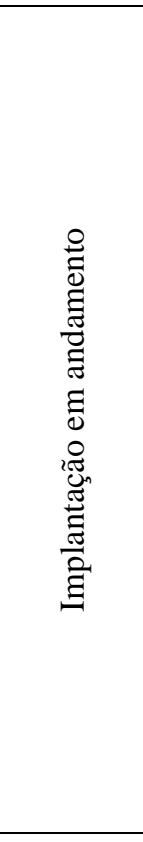 } & \multirow{4}{*}{$\begin{array}{l}\text { Motivação } \\
\text { do processo }\end{array}$} & 4 & $\begin{array}{l}\text { Alinhamento de métodos e técnicas e garantia da uniformidade nos } \\
\text { procedimentos. }\end{array}$ \\
\hline & & 5 & $\begin{array}{l}\text { Aumento de qualidade nos processos de fiscalização e credibilidade aos } \\
\text { usuários. }\end{array}$ \\
\hline & & 2 & O aprimoramento do controle externo e a adesão ao movimento mundial. \\
\hline & & 1 & Participação de projetos institucionais do sistema do Tribunal de Contas. \\
\hline & \multirow{4}{*}{$\begin{array}{l}\text { Recursos } \\
\text { humanos }\end{array}$} & 5 & De 1 a 5 funcionários. \\
\hline & & 2 & De 6 a 10 funcionários. \\
\hline & & 3 & Acima de 10 funcionários. \\
\hline & & 2 & Não responderam. \\
\hline & \multirow{3}{*}{$\begin{array}{l}\text { Recursos } \\
\text { financeiros }\end{array}$} & 7 & Não foi utilizado nenhum recurso financeiro no processo. \\
\hline & & 1 & Não houve utilização de recurso financeiro, mas poderá ocorrer. \\
\hline & & 4 & $\begin{array}{l}\text { Dispêndios com consultorias, viagens para capacitação e redesenho do } \\
\text { processo. }\end{array}$ \\
\hline & \multirow{2}{*}{$\begin{array}{l}\text { Normativo } \\
\text { específico }\end{array}$} & 3 & $\begin{array}{l}\text { Não possuem: (1) aguardando a conclusão da implantação; (2) sendo } \\
\text { estudado. }\end{array}$ \\
\hline & & 9 & Possuem normativa. \\
\hline & \multirow{3}{*}{$\begin{array}{l}\text { Prazo para } \\
\text { conclusão }\end{array}$} & 1 & Final de 2020. \\
\hline & & 1 & Final de 2021. \\
\hline & & 10 & Não informaram. \\
\hline \multirow{8}{*}{$\begin{array}{l}\frac{0}{0} \\
\frac{0}{0} \\
0 \\
0\end{array}$} & $\begin{array}{l}\text { Motivação } \\
\text { do processo }\end{array}$ & 2 & Importância de padronização para a qualidade dos trabalhos. \\
\hline & \multirow{2}{*}{$\begin{array}{l}\text { Recursos } \\
\text { humanos }\end{array}$} & 1 & Todos os auditores em atividade (não informou a quantidade). \\
\hline & & 1 & 6 auditores. \\
\hline & \multirow{2}{*}{$\begin{array}{l}\text { Recursos } \\
\text { financeiros }\end{array}$} & 1 & Não foram utilizados recursos financeiros no processo. \\
\hline & & 1 & Não respondeu. \\
\hline & $\begin{array}{l}\text { Normativo } \\
\text { específico }\end{array}$ & 2 & Possuem normativa. \\
\hline & \multirow{2}{*}{$\begin{array}{c}\text { Prazo de } \\
\text { implantação }\end{array}$} & 1 & 2 anos de duração. \\
\hline & & 1 & 3 anos de duração. \\
\hline
\end{tabular}

Fonte: elaborada com dados das respostas ao Q2 da pesquisa.

Para a questão sobre quais normas já foram implantadas pelos TCs, apresenta-se a Tabela 3, na qual se indica a situação de cada uma das NBASP e das ISSAIs não convergidas, oriunda da questão fechada em que o respondente deveria marcar a opção referente a cada uma das normas. Pelo elencado na Tabela 3, verifica-se que a NBASP 20, que trata da transparência e do accountability, e a NBASP 30, que trata da gestão da ética, foram as mais implantadas. O resultado pode indicar a preocupação dos TCs com a prestação de contas. No entanto observou-se alto 
volume de órgãos que não cooperaram com essa questão, confirmando o estudo de Macagnan, Laureano, Rosso e Wartchow (2019), que citam um comportamento de transparência e de accountability não exemplar por parte dos TCs, por conseguinte, acarretando deficiência de governança pública, além das polêmicas advindas das práticas contestadas em suas atuações (Lino \& Aquino, 2020). Essas deficiências não contribuem para a confiança da sociedade quanto aos TCs, tampouco com a de outros países que possam vir a realizar transações com o Brasil, pontos aos quais as normas internacionais virão colaborar.

Tabela 3

Situação da implantação das normas em cada Tribunal de Contas

\begin{tabular}{|c|c|c|c|c|c|c|}
\hline \multicolumn{2}{|c|}{$\begin{array}{c}\text { Normas convergidas no Brasil } \\
\text { (NBASP) }\end{array}$} & \multicolumn{5}{|c|}{ Situação } \\
\hline $\begin{array}{l}\text { NBASP } \\
\text { (convergidas) }\end{array}$ & $\begin{array}{l}\text { ISSAIs } \\
\text { (originais) }\end{array}$ & $\begin{array}{c}\text { Não } \\
\text { iniciada }\end{array}$ & $\begin{array}{c}\text { Em } \\
\text { andamento }\end{array}$ & Implantada & $\begin{array}{c}\text { Sem } \\
\text { resposta }\end{array}$ & Total \\
\hline NBASP 10 & ISSAI 10 & & 3 & 3 & 6 & 12 \\
\hline NBASP 12 & ISSAI 12 & & 5 & 1 & 6 & 12 \\
\hline NBASP 20 & ISSAI 20 & & 3 & 4 & 5 & 12 \\
\hline NBASP 30 & ISSAI 30 & & 2 & 5 & 5 & 12 \\
\hline NBASP 40 & ISSAI 40 & 2 & 2 & 3 & 5 & 12 \\
\hline NBASP 100 & ISSAI 100 & & 6 & 3 & 3 & 12 \\
\hline NBASP 200 & ISSAI 200 & 1 & 6 & 2 & 3 & 12 \\
\hline NBASP 300 & ISSAI 300 & & 6 & 3 & 3 & 12 \\
\hline NBASP 400 & ISSAI 400 & 1 & 5 & 3 & 3 & 12 \\
\hline NBASP 3000 & ISSAI 3000 & & 5 & 2 & 5 & 12 \\
\hline NBASP 4000 & ISSAI 4000 & 1 & 4 & 2 & 5 & 12 \\
\hline \multicolumn{2}{|c|}{$\begin{array}{c}\text { Normas não convergidas no } \\
\text { Brasil }\end{array}$} & $\begin{array}{c}\text { Não } \\
\text { iniciada }\end{array}$ & $\begin{array}{c}\text { Em } \\
\text { andamento }\end{array}$ & Implantada & $\begin{array}{c}\text { Sem } \\
\text { resposta }\end{array}$ & Total \\
\hline \multicolumn{2}{|c|}{ ISSAI 1} & & 2 & 2 & 8 & 12 \\
\hline \multicolumn{2}{|l|}{ ISSAI 11} & 1 & 2 & 2 & 7 & 12 \\
\hline \multicolumn{2}{|l|}{ ISSAI 21} & 1 & 3 & 2 & 6 & 12 \\
\hline \multicolumn{2}{|l|}{ ISSAI 3100} & 2 & 3 & 1 & 6 & 12 \\
\hline \multicolumn{2}{|c|}{ Apêndice ISSAI 3100} & 2 & 3 & 1 & 6 & 12 \\
\hline \multicolumn{2}{|l|}{ ISSAI 3200} & 2 & 3 & 1 & 6 & 12 \\
\hline
\end{tabular}

Fonte: elaborada com dados das respostas ao Q2 da pesquisa.

Também se destacaram as NBASP 100, 200, 300, 400 e 3000, que tratam do financeiro e do operacional do processo de auditoria, que pareceram mais próximas de serem concluídas e tendem a qualificar a atuação dos órgãos. Em relação às normas ainda não convergidas no Brasil, pode-se verificar, na Tabela 3, que alguns TCs as observavam ou estavam trabalhando para sua utilização, a despeito de não estarem expressamente inclusas no movimento nacional de adoção dos padrões.

\subsection{Melhorias com a implantação das normas internacionais de auditoria (NBASP)}

Esta subseção expõe os resultados com relação às melhorias que a implantação das NBASP pode oferecer ao Brasil perante outros países e a sociedade. Na Tabela 4, constam as melhorias citadas pelas instituições apoiadoras $(\mathrm{Q} 1)$ e TCs $(\mathrm{Q} 2)$ respondentes. 
Tabela 4

Melhorias descritas pelas instituições apoiadoras e tribunais

\begin{tabular}{|c|c|c|c|}
\hline Questões & $\begin{array}{l}\text { Alvo das } \\
\text { melhorias }\end{array}$ & $\begin{array}{l}\text { N. }{ }^{\circ} \text { de } \\
\text { respostas }\end{array}$ & Melhorias apontadas pelas instituições \\
\hline \multirow{7}{*}{ 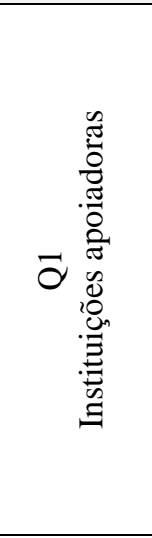 } & \multirow{5}{*}{$\begin{array}{l}\text { Brasil perante } \\
\text { outros países }\end{array}$} & 1 & Credibilidade tanto para os órgãos de controle como para os governos. \\
\hline & & 1 & $\begin{array}{l}\text { Reconhecimento e melhora da imagem do país em âmbito } \\
\text { internacional, a transparência e a fidedignidade das informações } \\
\text { financeiras divulgadas. }\end{array}$ \\
\hline & & 1 & $\begin{array}{l}\text { Permitirá o intercâmbio de informações, asseguração de relatórios e } \\
\text { aplicação de técnicas fiscais alinhadas com as práticas internacionais. }\end{array}$ \\
\hline & & 2 & $\begin{array}{l}\text { Sinaliza um futuro de mais segurança jurídica para fiscalizador e } \\
\text { fiscalizado. }\end{array}$ \\
\hline & & 1 & Não respondeu. \\
\hline & \multirow[t]{2}{*}{ Sociedade } & 4 & $\begin{array}{l}\text { Garantia de recursos aplicados de forma econômica, eficiente, efetiva, } \\
\text { em conformidade com leis e regulamentos e informações livres de } \\
\text { distorções. }\end{array}$ \\
\hline & & 2 & Não respondeu. \\
\hline \multirow{2}{*}{ 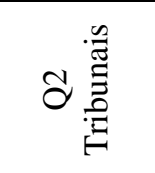 } & \multirow{2}{*}{$\begin{array}{l}\text { Brasil perante } \\
\text { outros países } \\
\text { e sociedade }\end{array}$} & 10 & $\begin{array}{l}\text { Aumento da credibilidade e qualidade dos trabalhos, profissionalismo } \\
\text { nas fiscalizações, fortalecimento institucional do controle externo e } \\
\text { otimização do tempo de realização das auditorias. }\end{array}$ \\
\hline & & 7 & Não responderam. \\
\hline
\end{tabular}

Fonte: elaborada com dados das respostas aos questionários da pesquisa.

As respostas encontradas revelaram-se de acordo com estudos sobre o tema, demonstraram ciência dos órgãos quanto à importância da implantação das normas internacionais de auditoria para a qualificação da gestão pública e assinalaram a relevância e credibilidade aos stakeholders (Gørrissen, 2020; Hay \& Cordery; 2021, Dragustin \& Pitulice, 2021). Em relação ao Q2, a questão sobre as melhorias foi efetuada somente aos 17 TCs que apresentaram as situações: $i$ ) estudada; $i$ ) em andamento e iii) concluída. O reconhecimento da importância da adoção das NBASP está alinhado com as motivações apresentadas para o início do processo de adoção das normas.

\subsection{Dificuldades nos processos de implantação das NBASP}

Buscou-se verificar as principais dificuldades na implantação das NBASP. Com relação aos TCs (20), algumas dificuldades foram apontadas (Tabela 5).

\section{Tabela 5}

\section{Dificuldades encontradas pelos tribunais para implantação das normas}

\begin{tabular}{c|l}
\hline $\begin{array}{c}\text { N. } \\
\text { resposta }\end{array}$ & Dificuldades encontradas pelos tribunais \\
\hline 6 & Resistência dos profissionais à mudança nos padrões da auditoria. \\
\hline 6 & $\begin{array}{l}\text { Recursos humanos, reorganização das unidades administrativas e dos procedimentos internos e o } \\
\text { desconhecimento das NBASP por profissionais integrados ao processo. }\end{array}$ \\
\hline 2 & Não enfrentaram dificuldades relevantes durante o processo. \\
\hline
\end{tabular}

Fonte: elaborada com dados das respostas ao Q2 da pesquisa.

Os tribunais que não iniciaram, ou que estavam estudando o processo de implantação das NBASP, não foram questionados em razão de não terem iniciado o processo, tendo eles apontado dificuldades quando questionados sobre o motivo da não adesão às normas (Tabela 2).

Quanto às instituições apoiadoras, nenhuma informou objeções na adoção das normas. Foi destacado que ocorreu demora no início do processo, mas, com a edição das normas de nível 3 das NBASP (2019), muitos dos TCs estudaram ou até mesmo iniciaram o processo da adoção.

Destacaram-se desafios oriundos da resistência de profissionais mais experientes em relação a mudanças, além da carência de recursos humanos e sua capacitação, pontos importantes 
para a implantação destacados na literatura e condizentes com as diferentes práticas observadas, até mesmo, pelos auditores de único órgão (Azevedo \& Lino, 2018). O achado indica ponto importante a ser trabalhado para o sucesso do processo.

\subsection{Qualificação dos profissionais de auditoria}

A pesquisa verificou a necessidade de qualificação dos profissionais de auditoria, cujos resultados constam na Tabela 6. Tanto as instituições quanto os TCs demonstraram reconhecimento no que tange à importância da capacitação para o processo, relatando as participações em eventos e estudos. Subentende-se que os tribunais que não iniciaram o processo de implantação também não capacitaram seus profissionais.

\section{Tabela 6}

Necessidade de qualificação dos profissionais para as novas normas para as instituições

\begin{tabular}{|c|c|c|c|}
\hline & Setor & $\begin{array}{l}\text { N.o de } \\
\text { resposta }\end{array}$ & Qualificação dos profissionais \\
\hline \multirow{2}{*}{\multicolumn{2}{|c|}{$\begin{array}{l}\text { Instituições } \\
\text { Apoiadoras }\end{array}$}} & 4 & $\begin{array}{l}\text { Existência de comitês que participam de eventos e congressos promovidos } \\
\text { para a divulgação e estudo das NBASP. }\end{array}$ \\
\hline & & 2 & Não possuem informações sobre a capacitação e escolha de profissionais. \\
\hline \multirow{6}{*}{ 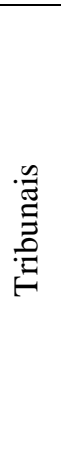 } & \multirow{2}{*}{$\begin{array}{l}\text { Estudando a } \\
\text { possibilidade de } \\
\text { implantar as } \\
\text { NBASP }\end{array}$} & 2 & $\begin{array}{l}\text { Serão realizados workshops, palestras, oficinas, treinamentos } \\
\text { colaborativos, aulas expositivas e distribuição de material sobre as } \\
\text { NBASP. }\end{array}$ \\
\hline & & 1 & Não houve capacitação nem nomeação de servidores para esse projeto. \\
\hline & \multirow{3}{*}{$\begin{array}{l}\text { Em processo de } \\
\text { implantação das } \\
\text { NBASP }\end{array}$} & 6 & $\begin{array}{l}\text { Palestras, fóruns, seminários promovidos pelo IRB e materiais } \\
\text { disponibilizados pelo órgão apoiador e emissor das NBASP. }\end{array}$ \\
\hline & & 4 & $\begin{array}{l}\text { Participação em cursos, grupos de discussões das normas e treinamentos } \\
\text { com convidados. }\end{array}$ \\
\hline & & 2 & Não responderam. \\
\hline & $\begin{array}{l}\text { Implantação } \\
\text { concluída }\end{array}$ & 2 & Cursos a distância e seminários presenciais. \\
\hline
\end{tabular}

Fonte: elaborada com dados das respostas aos questionários da pesquisa.

A relevância das organizações profissionais destacou-se nesse ponto (ReichbornKjennerud et al., 2019; Hay \& Cordery, 2020; Luz, Guarido, \& Sousa, 2021), visto que o IRB é bastante mencionado na promoção de eventos e publicações sobre o tema (IRB, 2020). A Abracom e a Audicon, que têm entre suas finalidades a promoção e o incentivo de eventos com a participação de especialistas, também se mostram relevantes no processo.

\section{CONSIDERAÇÕES FINAIS}

Este estudo foi realizado com o objetivo de analisar o processo de adoção das normas internacionais de auditoria do setor público nos Tribunais de Contas brasileiros. Entende-se que o objetivo foi atingido, pois foram identificados os obstáculos e os benefícios envolvidos, além de, com o presente, colaborar com uma base para pesquisas relativas ao tema. A análise do progresso da implantação das NBASP pelos TCs demonstrou que estão sendo empreendidos esforços nesse sentido, não obstante restarem distantes a utilização dos padrões por todos os órgãos e o consequente alcance dos benefícios à sociedade brasileira advindos das melhores práticas.

Entre os 33 Tribunais de Contas existentes no Brasil, somente 20 cooperaram com esta pesquisa, sendo essa uma de suas limitações. Verificou-se que apenas dois (10\%) TCs da amostra já concluíram a implantação das NBASP, 12 (60\%) estão em andamento e seis (30\%) ainda não iniciaram o processo. Os resultados reforçaram os relatos de pesquisas precedentes acerca das diferenças entre as práticas dos órgãos brasileiros (Azevedo \& Lino, 2018; Lino \& Aquino, 2018; 
Lino \& Aquino, 2020; Nunes, Marcelino, \& Silva, 2019; Luz, Guarido, \& Sousa, 2021) e em comparações internacionais (Manes-Rossi, Brusca, \& Condor, 2020; Cordery \& Hay, 2021).

O Brasil é um país de dimensões continentais, com grandes diferenças entre suas regiões, o que pode resultar em distintas práticas. Nesse sentido, porém, os achados quanto à vasta disponibilidade de capacitação, ao apoio fornecido pelas organizações e à ausência de necessidade de recursos financeiros pelos órgãos que concluíram o processo deixaram dúvidas concernentes às motivações dos TCs que não iniciaram o processo. A formalização da adoção das normas por meio de um normativo interno parece ser um ponto importante, dessa forma, destacando a relevância do interesse da gestão do órgão para o direcionamento de esforços à mudança dos procedimentos.

Conforme verificado, a adoção dos padrões internacionais de auditoria pelos TCs é reconhecida como importante para que haja melhorias nas parcerias internacionais e aumento da credibilidade e confiança da sociedade, o que é relevante diante dos episódios de corrupção identificados no país e das críticas por práticas socialmente contestadas por parte dos órgãos (Lino \& Aquino, 2020). Porém a falta de resposta à pesquisa por parte dos órgãos denota deficiência na aderência à legislação em vigor, pois a Lei n. ${ }^{\circ} 12.527$, de 18 de novembro de 2011 , estabelece a obrigação dos órgãos em repassar informações solicitadas pelo cidadão, e o não atendimento, por sua vez, contraria a legitimação esperada pela adoção das normas, assim, comprometendo os avanços nesse sentido, como a adoção das NBASP 20 e 30, que emanam os princípios da transparência e accountability com a gestão da ética, concluída por parte dos TCs.

Os resultados obtidos relataram, de forma geral, que os TCs brasileiros buscam melhorar a qualidade da fiscalização, visto que a maioria dos respondentes iniciou o processo de implantação das normas internacionais, e que há ações conjuntas implementadas por organizações de apoio. Contudo a falta de previsão de conclusão do processo pela maioria dos respondentes, bem como os comportamentos contraditórios verificados, indica haver um longo caminho pela frente. Para dirimir as diferentes práticas e assegurar a utilização de padrões para a melhoria das auditorias, a criação de um órgão de supervisão independente tem o potencial de contribuir nessa direção (Lino, Azevedo, Aquino, \& Steccolini, 2021).

Ressalta-se que os resultados desta pesquisa podem colaborar com o avanço do processo de implantação das normas internacionais de auditoria ao destacar a situação diversa e incipiente por muitos TCs, bem como por analisar pontos de apoio e obstáculos ao seu sucesso. Para trabalhos futuros, sugere-se analisar o fenômeno com apoio de lentes teóricas, estudar a aderência específica para cada uma das normas e investigar se, além da formalização da implantação das normas, a prática dos órgãos corresponde aos novos padrões.

\section{REFERÊNCIAS}

Azevedo, R. R., \& Lino, A. F. (2018). O distanciamento entre as normas de auditoria e as práticas nos Tribunais de Contas. Sociedade, Contabilidade e Gestão, 13(2), 9-27. Recuperado de http://dx.doi.org/10.21446/scg_ufrj.v13i2.13665

Azuma, N. (2008). The framework of INTOSAI Government Auditing Standards: in the stream of international convergence. Government Auditing Review, 15(1), 77-97. Recuperado de https://www.jbaudit.go.jp/english/exchange/pdf/e15d05.pdf

Bardin, L. (2011). Análise de conteúdo (L. A. Reto, \& A. Pinheiro, Trad.; 70. ed.). São Paulo: Edições 70.

Caruana, J., \& Kowalczyk, M. (2021). The quest for audit quality in the public sector. Accounting in Europe, 18(1), 1-25. Recuperado de http://dx.doi.org/10.1080/17449480.2020.1757731 
Cordery, C. J., \& Hay, D. C. (2021). Public sector audit in uncertain times. Financial Accountability \& Management. Recuperado de https://doi.org/10.1111/faam.12299

Dragustin, C., Pitulice, I., \& Stefanescu, A. (2021). Harmonisation and emergence concerning the performance audit of the EU member states' public sector. Romania's case. Sustainability, 13(7), 3673. Recuperado de http://dx.doi.org/10.3390/su1307367

Gørrissen, E. (2020). The role of the INTOSAI Development Initiative (IDI) in strengthening the capacity and performance of supreme audit institutions in developing countries. Journal of Public Budgeting, Accounting \& Financial Management, 32(4), 729-733. Recuperado de https://doi.org/10.1108/JPBAFM-08-2020-0146

Gustavson, M., \& Rothstein, B. (2013). Can auditing generate trust? The organization of auditing and the quality of government. In M. Reuter, F. Wijkstrom, B. Uggla (Eds.). Trust and organizations (pp. 41-63). New York: Palgrave Macmillan. Recuperado de https://doi.org/10.1057/9781137368812_3

Hay, D., \& Cordery, C. (2021) Evidence about the value of financial statement audit in the public sector. Public Money \& Management, 41(4), 304-314. Recuperado de https://doi.org/10.1080/09540962.2020.1729532

Hay, D., \& Cordery, C. (2020). The future of auditing research in the public sector. Journal of Public Budgeting, Accounting \& Financial Management, 33(2), 234-242. Recuperado de https://doi.org/10.1108/JPBAFM-09-2020-0164

INTOSAI - International Organisation of Supreme Audit Institutions (2021). Recuperado de https://www.intosai.org/

IRB - Instituto Rui Barbosa. (2020). Normas Brasileiras de Auditoria no Setor Público (NBASP): nível 1: princípios basilares pré-requisitos para o funcionamento dos Tribunais de Contas brasileiros. Recuperado de https://irbcontas.org.br/biblioteca/nbasp-1/

IRB - Instituto Rui Barbosa. (2019). Normas Brasileiras de Auditoria do Setor Público (NBASP): nível 3: requisitos mandatórios para auditorias do setor público. Recuperado de https://irbcontas.org.br/nbasp/

IRB - Instituto Rui Barbosa. (2020). Sobre o IRB. Recuperado de https://irbcontas.org.br/sobre-oirb/

Kontogeorga, G. N. (2019). Juggling between ex-ante and ex-post audit in Greece: A difficult transition to a new era. International Journal of Auditing, 23(1), 86-94. Recuperado de https://doi.org/10.1111/ijau.12147

Lino, A. F., \& Aquino, A. C. B. (2018). A diversidade dos Tribunais de Contas regionais na auditoria de governos. Revista Contabilidade \& Finanças, 29(76), 26-40. Recuperado de https://doi.org/10.1590/1808-057x201803640

Lino, A. F., \& Aquino, A. C. B. (2020). Práticas não adequadas nos Tribunais de Contas. Revista de Administração Pública, 54(1), 220-242. Recuperado de https://doi.org/10.1590/0034761220190270 
Lino, A. F., Azevedo, R. R., Aquino, A. C. B., \& Steccolini, I. (2021). Fighting or supporting corruption? The role of public sector audit organizations in Brazil. Critical Perspectives on Accounting, 8, 102384. Recuperado de https://doi.org/10.1016/j.cpa.2021.102384

López-Hernández, A. (2013). Normas profesionales de la INTOSAI: directrices de auditoría. Revista Auditoría Pública, 61(1), 9-24. Recuperado de https://asocex.es/wpcontent/uploads/PDF/Pag\%209-24\%20N\%C2\%BA\%2061.pdf

Luz, B. B. C., Guarido, E. R. F., \& Sousa, M. M. (2021). Legal, normativo e responsivo: frames no debate sobre a reforma dos Tribunais de Contas brasileiros. Revista de Administração Pública, 55(4), 861-880. Recuperado de https://bit.ly/395ogvj

Macagnan, C. B, Laureano, R. V, Rosso, V. P., \& Wartchow, D. (2020). Tribunais de Contas brasileiros são transparentes. Cuadernos de Contabilidad, 20(50). Recuperado de https://doi.org/10.11144/Javeriana.cc20-50.tcbt

Manes-Rossi, F., Brusca, I., \& Condor, V. (2020): In the pursuit of harmonization: comparing the audit systems of European local governments. Public Money \& Management, 1-11. Recuperado de https://doi.org/10.1080/09540962.2020.1772549

Nunes, S. P., Marcelino, G. F., \& Silva, C. A. T. (2019). Os Tribunais de Contas na interpretação da Lei de Responsabilidade Fiscal. Revista de Contabilidade e Organizações, 13(1), 1-15. Recuperado de https://doi.org/10.11606/issn.1982-6486.rco.2019.145151

Reichborn-Kjennerud, K., González-Díaz, B., Bracci, E., Carrington, T., Hathaway, J., Jeppesen, K. K, \& Steccolini, I. (2019). Sais work against corruption in Scandinavian, South-European and African countries: an institutional analysis. The British Accounting Review, 51(5), 100842. Recuperado de https://doi.org/10.1016/j.bar.2019.100842

STN - Secretaria do Tesouro Nacional. (2018). Manual de Contabilidade Aplicada ao Setor Público. https://sisweb.tesouro.gov.br/apex/f?p=2501:9::::9:P9_ID_PUBLICACAO:31484

Speck, B. W. (2000). Inovação e rotina no Tribunal de Contas da União: o papel da instituição superior de controle financeiro no sistema político-administrativo do Brasil. São Paulo: Fundação Konrad Adenauer.

TCU - Tribunal de Contas da União. (2020a) Normas Brasileiras de Auditoria no Setor Público (NBASP). Recuperado de https://portal.tcu.gov.br/fiscalizacao-e-controle/auditoria/normasbrasileiras-de-auditoria-do-setor-publico-nbasp/

TCU - Tribunal de Contas da União. (2020b). Normas Internacionais das Entidades Fiscalizadoras Superiores (ISSAI) - ISSAI em português. Recuperado de https://portal.tcu.gov.br/fiscalizacao-e-controle/auditoria/normas-internacionais-dasentidades-fiscalizadores-superiores-issai/ 